\title{
Axiomatic Characterization of the Antimedian Function on Paths and Hypercubes
}

\author{
Kannan Balakrishnan \\ Department of Computer Applications, Cochin University of Science and Technology \\ Cochin - 682 022, India \\ e-mail: bkannan@cusat.ac.in \\ Manoj Changat* \\ Department of Futures Studies, University of Kerala \\ Trivandrum - 695 034, India \\ e-mail: mchangat@gmail.com \\ Henry Martyn Mulder ${ }^{\dagger}$ \\ Econometrisch Instituut, Erasmus Universiteit \\ P.O. Box 1738, 3000 DR Rotterdam, Netherlands. \\ e-mail:hmmulder@few.eur.nl \\ Ajitha R. Subhamathi \\ Department of Computer Applications \\ N.S.S College Rajakumari, Idukki, Kerala, India \\ Trivandrum - 695 034, India \\ e-mail:ar.subhamathi@gmail.com
}

4 March 2011

Econometric Institute Report EI 2011-08

This Report is a preprint. It is not to be considered a formal publication in any way. It will be submitted elsewhere.

${ }^{*}$ Research work is supported by NBHM/DAE under grant No.2/48(2)/2010/NBHM-R \& D

${ }^{\dagger}$ This research was carried out while the third author visited the University of Kerala in January 2011 under the Erudite Scheme of the Government of Kerala, India. 


\begin{abstract}
An antimedian of a profile $\pi=\left(x_{1}, x_{2}, \ldots, x_{k}\right)$ of vertices of a graph $G$ is a vertex maximizing the sum of the distances to the elements of the profile. The antimedian function is defined on the set of all profiles on $G$ and has as output the set of antimedians of a profile. It is a typical location function for finding a location for an obnoxious facility. The 'converse' of the antimedian function is the median function, where the distance sum is minimized. The median function is well studied. For instance it has been characterized axiomatically by three simple axioms on median graphs. The median function behaves nicely on many classes of graphs. In contrast the antimedian function does not have a nice behavior on most classes. So a nice axiomatic characterization may not be expected. In this paper such a characterization is obtained for the two classes of graphs on which the antimedian is well-behaved: paths and hypercubes.
\end{abstract}

Keywords: Antimedian, consensus function, consistency, path, hypercube, consensus axiom

AMS subject classification (2000): 05C12, 05C99, 05C38, 90B 80

\title{
1 Introduction
}

The general problem in discrete location theory deals with functions that find a site on a graph in such a way as to minimize some cost, or maximize some benefit, to a given set of clients represented by vertices on the graph. Typical problems of this kind that have been studied extensively are: 1 . the median problem: finding a vertex that minimizes the distance sum to the clients; 2 . the mean problem: finding a vertex that minimizes the sum of the squares of the distances to the clients; 3 . the center problem: minimizing the maximum distance to clients. The first two problems could be used to model finding the optimal location for a distribution center. The last problem could be used to model finding the optimal location for a fire station. In this paper we focus on a different type of location problem. Now the facility is one that is of obnoxious nature: the clients want to have it as far away as possible, for example a garbage dump.

Location problems can be phrased as consensus problems. Then one wants to reach consensus amongst agents or clients in a rational way. This is modelled using a consensus function. The input of the function consists of certain information about the agents, and the output concerns the issue about which consensus should be reached. The rationality of the process is guaranteed by the fact that the consensus function satisfies certain "rational" rules or "consensus axioms". Such consensus axioms should be appealing and simple.

From this point of view one wants to characterize a location function by a set of a nice and simple axioms. Holzman [2] was the first to study location functions in this way. His focus was in the mean function on a tree network (the continuous 
variant of a tree). Then Vohra [13] characterized the median function axiomatically on tree networks (continuous case). The discrete case was first dealt with by McMorris, Mulder \& Roberts [5]: the median function on cube-free median graphs using three simple and appealing axioms, see below. The mean function on trees (discrete case) was first characterized by McMorris, Mulder \& Ortega [4]. The center function on trees has been characterized by McMorris, Roberts \& Wang [7], see also [12]. Recently the median function was characterized on hypercubes by Mulder \& Novick [11] using the same three simple axioms as in [5]. In the case of the median function and the center function all axioms satisfy the criterion of being simple and natural at first sight. The axioms for the mean function are more complex than those for the median function or center function. But except for one they still satisfy the criterion of being simple and appealing. All above characterizations for the center function and the mean function so far are on trees. The characterization for the median function is on a much wider class, viz. that of median graphs. The reason for this is the very nice behavior of the median function on these graphs.

The focus of this paper is the first characterization of an obnoxious location function. It is the antimedian function, which maximizes the sum of the distances to the clients. At first sight it looks very similar to the median function. But the differences are quite striking. A first inspection of the antimedian function already shows that, even on trees, it does not behave nicely at all, let alone on arbitrary graphs. Only on paths and hypercubes it has a nice behavior. So one may expect characterizations by simple and appealing axioms on these two classes of graphs. This is the aim of the paper. In Section 2 we set the stage. In Section 3 we characterize the antimedian function on paths by a set of six axioms. They are all simple and natural. We show that these are independent. Finally, in Section 5 we use the theory of the median function to arrive simply at a characterization the antimedian function on hypercubes.

\section{Preliminaries}

Let $G=(V, E)$ be a finite, connected, simple graph with vertex set $V$ and edge set $E$. The distance function of $G$ is denoted by $d$, where $d(u, v)$ is the length of a shortest $u, v$-path. The interval $I(u, v)$ between two vertices $u$ and $v$ in $G$ consists of all vertices on shortest $u, v$-paths, that is:

$$
I(u, v)=\{x \mid d(u, x)+d(x, v)=d(u, v)\} .
$$

A profile $\pi$ of length $k=|\pi|$ on $G$ is a sequence $\pi=\left(x_{1}, x_{2}, \ldots, x_{k}\right)$ of vertices of $V$ with repetitions allowed. We define $V^{*}$ to be the set of all profiles of finite length. The concatenation of profiles $\pi$ and $\rho$ is denoted by $\pi \rho$. The profile consisting of the concatenation of $m$ copies of $\pi$ is denoted by $\pi^{m}$. The profile $(x)^{m}=(x, x, \ldots, x)$ consisting of $m$ copies of $x$ is called a constant profile. So a non-constant profile contains 
at least two distinct elements. A subprofile of $\pi$ is obtained by deleting elements from $\pi$. If we say that $x$ is an element of $\pi$, denoted by $x \in \pi$, then we mean an element on a certain position, say $x=x_{i}$ in the $i$-th position. Then $\pi-x$ denotes the subprofile obtained by deleting the $i$-th element from $\pi$. So, if vertex $x$ occurs more than once in $\pi$, we only delete $x$ from the $i$-th place in $\pi$, and leave the other occurrences of $x$ in $\pi$ untouched. We follow the same usage when we delete more elements from $\pi$. So, for a subprofile $\pi^{\prime}$ of $\pi$, the profile $\pi-\pi^{\prime}$ is the subprofile of $\pi$ obtained by deleting all elements of $\pi^{\prime}$ from $\pi$.

A consensus function on $G$ is a function $F: V^{*} \rightarrow 2^{V}-\emptyset$ that gives a non-empty subset as output for each profile on $G$. For convenience, we will write $F\left(x_{1}, \ldots, x_{k}\right)$ instead of $F\left(\left(x_{1}, \ldots, x_{k}\right)\right)$, for any function $F$ defined on profiles, but will keep the brackets where needed.

The remoteness of a vertex $v$ to profile $\pi$ is defined as

$$
r(v, \pi)=\sum_{i=1}^{k} d\left(x_{i}, v\right) .
$$

A vertex minimizing $r(v, \pi)$ is called a median of the profile. The set of all medians of $\pi$ is the median set of $\pi$ and is denoted by $M(\pi)$. A vertex maximizing $r(v, \pi)$ is called an antimedian of the profile. The set of all antimedians of $\pi$ is the antimedian set of $\pi$ and is denoted by $A M(\pi)$. We also think of $M$ and $A M$ as functions from $V^{*}$ to $2^{V}-\emptyset$, and then call them the Median Function and Antimedian Function. Note that we have $M(x)=\{x\}$, and $M(x, y)=I(x, y)$. Moreover, if $I(u, v) \cap I(v, w) \cap I(w, u) \neq \emptyset$, then $M(u, v, w)=I(u, v) \cap I(v, w) \cap I(w, u)$.

The median function has been studied widely, especially on median graphs. A median graph is defined by the property that $|I(u, v) \cap I(v, w) \cap I(w, u)|=1$, for any three vertices $u, v, w$. See e.g. $[8,3,10]$ for a rich structure theory on median graphs. Also nice axiomatic characterizations are available for the median function on median graphs, see e.g. $[5,6,11]$. Three simple and natural axioms suffice for the characterization of the median function. We present these here. The first two axioms are defined without any reference to metric.

(A) Anonymity: $F(\pi)=F\left(x_{\chi(1)}, x_{\chi(2)}, \ldots, x_{\chi(k)}\right)$, for any profile $\pi=\left(x_{1}, x_{2}, \ldots, x_{k}\right)$ on $V$ and for any permutation $\chi$ of $\{1,2, \ldots, k\}$.

(C) Consistency: If $F(\pi) \cap F(\rho) \neq \emptyset$ for profiles $\pi$ and $\rho$, then $F(\pi \rho)=F(\pi) \cap F(\rho)$.

(B) Betweenness: $F(u, v)=I(u, v)$, for all $u, v \in V$.

Anonymity is a desirable axiom for many consensus problems. Consistency means that, if profile $\pi$ and profile $\rho$ both agree on the same output $x$, then the concatenated profile $\pi \rho$ should also agree on $x$, again a very natural axiom. The last axiom is the 
only axiom that involves the metric of the graph. There exist simple examples to show that $(B)$ and $(C)$ are independent, see e.g. [11].

Consistency implies that $F(\pi)=F\left(\pi^{m}\right)$, for any profile $\pi$ and any positive integer $m$. Anonymity means that we can rewrite a profile in any order that we find convenient. Because anonymity is presupposed for all functions considered, we will consider two profiles to be the same if the one can be obtained form the other by a permutation. For instance, $(0, p)^{m}$ denotes the profile of length $2 m$ containing 0 and $p$ both $m$ times (in any order).

It is clear from the extensive literature that the median function behaves very nicely on many classes of graphs. The antimedian function on the other hand is a function that behaves "badly": there is not much structure to be found in the family of antimedian sets of profiles. The antimedian function satisfies betweenness only on $K_{1}$. Being defined by a sum of distance, it clearly satisfies anonymity. It also satisfies consistency. The proof of this fact is almost identical to the proof of the consistency of the median function. For the sake of completeness, we include the proof here. We phrase it in terms of graphs, but the proof is the same for any finite metric space.

Lemma 1 Let $G=(V, E)$ be a connected graph. The antimedian function on $G$ is consistent.

Proof. Let $\pi$ and $\rho$ be profiles on $G$. Take any $x$ in $A M(\pi) \cap A M(\rho)$ and $y$ in $A M(\pi \rho)$. Then we have

$$
\begin{array}{rlrl}
r(y, \pi \rho) & =r(y, \pi)+r(y, \rho) \quad & \text { (by the definition of } r) \\
& \leq r(x, \pi)+r(x, \rho) \quad(\text { since } x \in A M(\pi) \cap A M(\rho) \\
& =r(x, \pi \rho) \quad(\text { by the definition of } r) \\
& \leq r(y, \pi \rho) \quad(\text { since } y \in A M(\pi \rho) .
\end{array}
$$

So we have equality everywhere, whence $x$ is in $A M(\pi \rho)$ and $y$ in $A M(\pi) \cap A M(\rho)$.

Consistency brings some structure to a consensus function $F$, but not yet much. A first inspection shows that even on trees one cannot expect much structure for the antimedian function, not to mention other less simple classes of graphs. So, as a first step, we consider two very well-structured classes: the paths and the hypercubes. They are both median graphs. Loosely speaking, they are the two 'opposites' within this class: the 'thinnest' ones and the 'thickets' ones.

Recall that a central vertex of a graph minimizes the maximum distance to any other vertex in the graph. The center is the set of all central vertices of the graph. The center of an even path consists of the unique middle vertex, and of an odd path it consists of the two middle vertices. 


\section{Axiomatic Characterization of the Antimedian Function on Paths}

In this Section we characterize the antimedian function on paths. For convenience, the path will be denoted by $P=0 \rightarrow 1 \rightarrow \cdots \rightarrow p$. So it is of length $p$ and has $V=\{0,1, \ldots, p\}$ as vertex set. Let $\psi$ denote the center of $P$. Note that $\psi=\{k\}$ in case $p=2 k$, and $\psi=\{k, k+1\}$ in case $p=2 k+1$, with $k$ a positive integer. We view the path as going from left to right. We call the set of all $x$ such that $x<\frac{1}{2} p$ the left half $L$ of $P$, similarly the set of vertices $x>\frac{1}{2} p$ is the right half $R$ of $P$. Note that, if $p=2 k$, then the center vertex $k$ of $P$ is excluded when considering the left half or right half of $P$. If $p=2 k+1$, then we call $k$ the left center $c_{L}$ and $k+1$ the right center $c_{R}$. If $p=2 k$, then we call $k$ the left center $c_{L}$ as well as the right center $c_{R}$. This 'abuse' of terminology allows us to make statements where we do not need to distinguish between the even and odd case. For any profile $\pi$ on $P$ and any vertex $i$ of $P$, we denote by $\pi_{\leq i}$ the subprofile of $\pi$ consisting of all elements $x$ of $\pi$ with $x \leq i$, and we write $\pi_{>i}=\pi-\pi_{\leq i}$. Similarly, $\pi_{\geq i}$ is the subprofile consisting of all elements $y$ of $\pi$ with $y \geq i$. First we study the antimedian sets in $P$.

Lemma 2 Let $\pi$ be a profile on $P$, and let $i$ be a vertex on $P$ with $0 \leq i<p$. Then

(i) $r(i, \pi)>r(i+1, \pi)$ if and only if $\left|\pi_{\leq i}\right|<\left|\pi_{>i}\right|$,

(ii) $r(i, \pi)<r(i+1, \pi)$ if and only if $\left|\pi_{\leq i}\right|>\left|\pi_{>i}\right|$.

Proof. By the definition of remoteness, we have

$$
\begin{aligned}
r(i, \pi) & =r\left(i, \pi_{\leq i}\right)+r\left(i, \pi_{>i}\right) \\
& =r\left(i+1, \pi_{\leq i}\right)-\left|\pi_{\leq i}\right|+r\left(i+1, \pi_{>i}\right)+\left|\pi_{>i}\right| \\
& =r(i+1, \pi)+\left|\pi_{>i}\right|-\left|\pi_{\leq i}\right|,
\end{aligned}
$$

from which the lemma follows.

Corollary 3 Let $\pi$ be a profile on $P$, and let $i$ be a vertex of $P$ with $0 \leq i<p$. Then $r(i, \pi)=r(i+1, \pi)$ if and only if $\left|\pi_{\leq i}\right|=\left|\pi_{>i}\right|$.

Lemma 4 Let $\pi$ be a profile on $P$. Then $A M(\pi)=\{0\}$, or $\{p\}$, or $\{0, p\}$, or $V$.

Proof. Let $i$ be a vertex of $P$. Define the function $f$ on $V$ by $f(i)=\left|\pi_{\leq i}\right|$. Then, going from left to right along $P$, the function $f$ is a non-decreasing function. Lemma 2 and Corollary 3 imply that, going from left to right along $P$, the function $r$ first is nonincreasing (when $f(i)<\frac{1}{2}|\pi|$ ), then may be constant for some time (when $f(i)=\frac{1}{2}|\pi|$ ), and then is non-decreasing (when $f(i)>\frac{1}{2}|\pi|$ ). So, either 0 or $p$ or both $0, p$ are contained in $A M(\pi)$. 
Assume that there is some $j$ in $A M(\pi)$ with $0<j<p$. By the above properties of $r$, we deduce that $0, \ldots, j$ and/or $j, \ldots, p$ is contained in $A M(\pi)$. So 1 or $p-1$ is in $A M(\pi)$. First assume that 1 is in $A M(\pi)$. Then $r(0, \pi)=r(1, \pi)$, so by Corollary 3 , we have $\left|\pi_{\leq 0}\right|=\left|\pi_{>0}\right|=\frac{1}{2}|\pi|$. Since $f(i)$ is non-decreasing, it follows that $\left|\pi_{\leq 0}\right| \leq\left|\pi_{\leq i}\right|$, for all $i$. So, from Lemma 2 and $f$ being non-decreasing, it follows that $r$ is non-decreasing on the whole of $P$. Since 0 is in $A M(\pi)$, it maximizes the remoteness. Hence it follows that $r(i, \pi)$ is constant on $P$, and $A M(\pi)=V$. A similar argument deals with the case $p-1$ in $A M(\pi)$.

An extreme profile is a profile consisting of $m$ copies of 0 and $m$ copies of $p$ with $m$ a positive integer.

Lemma $5 A M(\pi)=V$ if and only if $\pi$ is an extreme profile.

Proof. First let $\pi$ be an extreme profile. By anonymity, we may write $\pi=(0, p)^{m}$. Then $r(v, \pi)=m[d(0, v)+d(v, p)]=m \times p$, for any $v$ on $P$. So $A M(\pi)=V$.

Conversely, assume that $A M(\pi)=V$. Then the remoteness of all vertices is the same, in particular, it is the same for any two vertices $i$ and $i+1$ with $0 \leq i<p$. By Corollary 3 , we have $\left|\pi_{\leq i}\right|=\left|\pi_{>i}\right|$, for any $i$ with $0 \leq i<p$. So half of $\pi$ is to the left from $i$ and half of $\pi$ is strictly to the right from $i$, for any $i$ with $0 \leq i<p$. In particular this holds for $i=0$. Hence exactly half of the elements of $\pi$ is equal to 0 . It also holds for $i=p-1$. Hence exactly half of the elements $\pi$ is strictly to the right of $p-1$, that is, equal to $p$. So $\pi$ is an extreme profile.

A central profile is a profile consisting only of center vertices with $c_{L}$ and $c_{R}$ occurring an equal number of times, say $m$ with $m$ a positive integer. If $p=2 k$, then $\pi$ is the constant profile consisting of $m$ copies of $k$. If $p=2 k+1$, then $\pi$ consists of $m$ copies of $k$ and $m$ copies of $k+1$.

Lemma $6 A M(\pi)=\{0, p\}$, for any central profile $\pi$.

Proof. By anonymity we may assume that $\pi=\psi^{m}$. First let $p=2 k$. Then $r(i, \pi)=m \times|k-i|$. The maximum $m \times k$ is realized by 0 and $p$.

Next let $p=2 k+1$. Then $r(i, \pi)=m \times|k-i|+m \times|k+1-i|$. The maximum $m \times p$ is realized by 0 and $p$.

We call a profile $\pi$ balanced if $A M(\pi)=\{0, p\}$. So central profiles are balanced. But there are many more balanced profiles. For instance, the profile $(i, p-i)$ is balanced for any $i$. Below we will see that being balanced can also be phrased in terms of properties of the profile. We postpone the calculation of $A M(\pi)$ for profiles $\pi$ that are not extreme and not balanced.

Let $\pi$ be a profile on $P$. Let $\pi_{L}$ be the subprofile of $\pi$ consisting of the elements of $\pi$ in the left half of $P$, and $\pi_{R}$ the subprofile of the elements in the right half of $P$. 
The following axioms are all defined on paths only. It is not clear how to generalize these even to trees or cycles, not to mention other classes of graphs. On the other hand, they are nice, succinct, and simple. The first axiom only involves profiles of length 1. The second and third axiom both only involve one profile (of length one or two). From the above lemmas we see that the antimedian function satisfies the first three axioms. The fourth axiom involves profiles of length at least two, but still only two elements of the profile are affected.

(F1) Singleton: $F(x)= \begin{cases}0 & \text { for } x<\frac{1}{2} p \\ p & \text { for } x>\frac{1}{2} p\end{cases}$

(Ce) Center: $F(\psi)=\{0, p\}$.

(Ex) Extreme: $F(0, p)=V$.

Let $\pi$ contain two elements $x, y$ with $x<y$ and $\{x, y\} \neq\{0, p\}$. Note that this is equivalent to $\pi$ not being extreme and not being constant. Then $\sigma(\pi)$ is the profile obtained from $\pi$ by replacing the element $x$ by $x+1$ and the element $y$ by $y-1$. We say that $\sigma(\pi)$ is obtained by the shift $\sigma=(x, y) \rightarrow(x+1, y-1)$. Note that we do not apply shifts to the pair $0, p$. A shift applied to $(x, x+1)$ results in just permuting the two elements involved in the shift. So, due to anonymity, it is the 'same' profile. Therefore, we need not bother about shifts of this type.

(Sh) Shift: $F(\pi)=F(\sigma(\pi))$, for any non-extreme and non-constant profile $\pi$ and for any shift $\sigma$ of $\pi$.

Let $\pi$ be a profile, and let $\pi^{\prime}$ be a profile obtained from $\pi$ by consecutively applying a number of shifts. Let $\rho$ be any profile obtained by a permutation of $\pi^{\prime}$. Because we always assume anonymity, we say that $\pi$ can be shifted to $\rho$.

Theorem 7 Let $\pi$ be a non-extreme, non-constant profile on $P$, and let $\sigma$ be a shift. Then $A M(\pi)=A M(\sigma(\pi))$.

Proof. Since $\pi$ is non-constant and non-extreme, it contains a pair of distinct elements $x, y$ with $0 \leq x<y \leq p$ and $\{x, y\} \neq\{0, p\}$. Let $\sigma=(x, y) \rightarrow(x+1, y-1)$. If $y=x+1$, then $\sigma(\pi)=\pi$, and we are done. So we may assume that $x<y-1$. Let $\pi^{\prime}=\pi-(x, y)$ be the subprofile obtained from $\pi$ by deleting the two elements $x$ and $y$. Then, by anonymity, $\sigma(\pi)=\pi^{\prime}(x+1, y-1)$ is the concatenation of $\pi^{\prime}$ and $(x+1, y-1)$. For any $v$ on $P$ with $0 \leq v \leq x$ we have 


$$
\begin{aligned}
\sum_{u \in \pi} d(u, v) & =\sum_{u \in \pi^{\prime}} d(u, v)+d(x, v)+d(y, v) \\
& =\sum_{u \in \pi^{\prime}} d(u, v)+d(x, v)+1+d(y, v)-1 \\
& =\sum_{u \in \pi^{\prime}} d(u, v)+d(x+1, v)+d(y-1, v) \\
& =\sum_{u \in \sigma(\pi)} d(u, v) .
\end{aligned}
$$

So the remoteness of $v$, with $0 \leq v \leq x$, does not change by shifting. A similar computation shows that the remoteness of $v$, with $y \leq v \leq p$, does not change. Now take any $v$ with $x<v<y$. Then we have

$$
\begin{aligned}
\sum_{u \in \pi} d(u, v) & =\sum_{u \in \pi^{\prime}} d(u, v)+d(x, v)+d(y, v) \\
& =\sum_{u \in \pi^{\prime}} d(u, v)+d(x, v)-1+d(y, v)-1+2 \\
& =\sum_{u \in \pi^{\prime}} d(u, v)+d(x+1, v)+d(y-1, v)+2 \\
& =\sum_{u \in \sigma(\pi)} d(u, v)+2 .
\end{aligned}
$$

So the remoteness of $v$ decreases by shifting.

Recapitulating, the remoteness remains the same for $v$ with $0 \leq v \leq x$ or $y \leq v \leq p$, and the remoteness reduces by 2 for $v$ with $x<v<y$.

It remains to prove that no $v$ with $x<v<y$ is in $A M(\pi)$. By Lemma 4, vertex $v$ is in $A M(\pi)$ if and only if $A M(\pi)=V$. By Lemma 5, this is the case if and only if $\pi$ is an extreme profile. But $\pi$ contains two elements $x$ and $y$ with $\{x, y\} \neq\{0, p\}$. So indeed $v$ is not in $A M(\pi)$, so that, by Lemma $4, A M(\pi) \subseteq\{0, p\}$. This completes the proof of the theorem.

Theorem 8 Let $\pi$ be a profile on the path $P$. Then $\pi$ is balanced if and only if $\pi$ can be shifted to a central profile.

Proof. First assume that $\pi$ can be shifted to $\psi^{m}$ with $m$ a positive integer. By Theorem 7 and Lemma 6, we have $A M(\pi)=A M\left(\psi^{m}\right)=\{0, p\}$. So $\pi$ is balanced. 
Conversely, assume that $\pi$ is a balanced profile, so that $A M(\pi)=\{0, p\}$. Note that, by definition of balanced, $\pi$ is not an extreme profile. We consider the values $r\left(c_{L}, \pi_{L}\right)$ and $r\left(c_{R}, \pi_{R}\right)$.

If $\pi$ is a central profile, then nothing has to be proved. So assume that $\pi$ is not a central profile. We consider two cases.

Case 1. $r\left(c_{L}, \pi_{L}\right)=r\left(c_{R}, \pi_{R}\right)=0$.

Then all elements of $\pi$ are in $\psi$. If $p$ is even, then $\psi=\{k\}$, and $\pi$ is the constant profile with element $k$. So $\pi$ is a central profile, and no shifts are needed. If $p$ is odd, then, in order that $A M(\pi)=\{0, p\}$, both $k$ and $k+1$ have to appear an equal number of times in $\pi$. So again $\pi$ is a central profile, and no shifts are needed.

Case 2. $r\left(c_{L}, \pi_{L}\right)+r\left(c_{R}, \pi_{R}\right)>0$. Since $r\left(c_{L}, \pi_{L}\right)$, and $r\left(c_{R}, \pi_{R}\right)$ both are nonnegative integers, at least one of them must be positive. Without loss of generality we may assume that $r\left(c_{L}, \pi_{L}\right)>0$, so that there is an element $x$ in $\pi$ with $x<k$. If all elements of $\pi$ are on $L$, then

$$
\begin{array}{rlrl}
r(p, \pi) & =d(p, x)+r(p, \pi-x)=(p-x)+r(p, \pi-x) \\
& \geq(p-x)+(|\pi|-1) d\left(p, c_{L}\right) \quad & \text { (because } \pi \text { is on } L) \\
& >x+(|\pi|-1) d\left(p, c_{L}\right) & & \text { (because } \left.x<\frac{1}{2} p\right) \\
& \geq x+(|\pi|-1) d\left(0, c_{L}\right) & \\
& =d(0, x)+(|\pi|-1) d\left(0, c_{L}\right) & \\
& \geq d(0, x)+r(0, \pi-x)=r(0, \pi) & \quad \text { (because } \pi \text { is on } L) .
\end{array}
$$

This is in conflict with $A M(\pi)=\{0, p\}$. So there exists an element $y$ in $\pi$ that is on $R$, so $y \geq k+1$. Now we apply shift $\sigma=(x, y) \rightarrow(x+1, y-1)$. Note that $x+1 \leq c_{L}$. So $r\left(c_{L}, \sigma(\pi)_{L}\right)=r\left(c_{L}, \pi_{L}\right)-1$. If $y>c_{R}$, then we have $r\left(c_{R}, \sigma(\pi)_{R}\right)=r\left(c_{R}, \pi_{R}\right)-1$. If $y=c_{R}$, so that $p$ is odd, then $y$ is shifted to $c_{L}$. Now we have $r\left(c_{R}, \sigma(\pi)_{R}\right)=r\left(c_{R}, \pi_{R}\right)$. But in all cases, the value of $r\left(c_{L}, \pi_{L}\right)+r\left(c_{R}, \pi_{R}\right)$ is reduced by applying the shift. As long as this value is positive, we can find a shift that reduces this value. So, after a sufficient number of shifts we obtain a profile for which this value is 0 . Then we are in Case 1, and the proof of the Theorem is complete.

We can phrase the property of being balanced completely in terms of the profile. We do this in the next theorem. Because we do not need this theorem in the sequel, we omit the proof. It is straightforward anyway.

Theorem 9 Let $\pi$ be a profile on $P$. Then $\pi$ is balanced if and only if $r\left(c_{L}, \pi_{L}\right)=$ $r\left(c_{R}, \pi_{R}\right)$ and $r\left(c_{L}, \pi_{R}\right)=r\left(c_{R}, \pi_{L}\right)$.

We call a profile $\pi$ with $A M(\pi)=\{0\}$ or $\{p\}$ unbalanced. Let $\pi$ be an unbalanced profile. If $\pi_{>c_{R}}$ is empty, the we call $\pi$ a left wing profile. If $\pi_{<c_{L}}$ is empty, then we 
call $\pi$ a right wing profile. Let $\pi$ be an unbalanced profile with all its elements in $\psi$. If $p=2 k$, then $\pi$ is a constant profile on $k$, so that it is a central profile, and therefore balanced. So $p=2 k+1$. The only way that $\pi$ is unbalanced is that it contains one of the centers more than the other, say it contains $m_{1}$ copies of $c_{1}$ and $m_{2}$ copies of $c_{2}$ with $m_{1}<m_{2}$. Then we can write $\pi$ as $\psi^{m_{1}}\left(c_{1}\right)^{m_{2}-m_{1}}$. We call such profiles central unbalanced. If $c_{1}=c_{L}$, then $A M(\pi)=\{p\}$. We call such a profile a left wing profile as well. If $c_{1}=c_{R}$, then $A M(\pi)=\{0\}$. We call such a profile a right wing profile as well. Note that a left wing profile has the right most vertex $p$ as its unique antimedian vertex, and a right wing profile has the left most vertex 0 as its unique antimedian vertex.

Theorem 10 Let $\pi$ be an unbalanced profile on $P$, then $\pi$ can be shifted to a left wing profile or a right wing profile.

Proof. We may assume that $\pi$ is not a left wing or right wing profile. Then necessarily $\pi$ contains an element $x$ with $x<c_{L}$ as well as an element $y$ with $y>c_{R}$. So $r\left(c_{L}, \pi_{L}\right)+r\left(c_{R}, \pi_{R}\right)>0$. By applying the shift $\sigma=(x, y) \rightarrow(x+1, y-1)$ we reduce this value by 2 . Moreover, $x+1 \leq c_{L}$ and $y-1 \geq c_{R}$. So the number of elements in $\pi_{L}$ as well as in $\pi_{R}$ does not change. Continuing the process we arrive at a left wing or right wing profile.

Combining Theorems 10 and 7 we get:

Corollary 11 Let $\pi$ be a profile on $P$. Then $A M(\pi)=\{0\}$ if and only if $\pi$ can be shifted to a right wing profile, and $A M(\pi)=\{0\}$ if and only if $\pi$ can be shifted to a left wing profile.

We are now ready to prove the main result of this paper: the axiomatic characterization of the antimedian function on paths.

Theorem 12 Let $F$ be a consensus function on the path $P=0 \rightarrow 1 \rightarrow \cdots \rightarrow p$. Then $F$ is the antimedian function if and only if $F$ satisfies $(A),(C),(F 1),(C e),(E x)$ and $(S h)$.

Proof. First consider the antimedian function $A M$. Then, by definition, $A M$ satisfies anonymity, and by Lemma $1, A M$ satisfies consistency. Obviously, $A M$ satisfies $(F 1)$. By Lemma 6 , it satisfies $(C e)$, and by Lemma 5 , it satisfies $(E x)$. Finally, by Theorem 7 , it satisfies $(S h)$.

Conversely, let $F$ be a consensus function on $P$ satisfying $(A),(C),(F 1),(C e)$, $(E x)$ and $(S h)$.

First let $\pi$ be an extreme profile. Then, by $(A)$, we have $\pi=(0, p)^{m}$ with $m$ a positive integer. By $(C)$, we have $F(\pi)=F(0, p)$, and, by $(E x)$ and Lemma 5 , we have $F(0, p)=V=A M(\pi)$. 
Next let $\pi$ be a central profile. Then, by $(A)$, we have $\pi=\psi^{m}$ with $m$ a positive integer. By $(C)$, we have $F\left(\psi^{m}\right)=F(\psi)$, and, by $(C e)$ and Lemma 6 , we have $F(\psi)=\{0, p\}=A M(\pi)$.

Now let $\pi$ be a profile that is neither extreme nor central. If $\pi$ contains a pair of elements $x, y$ with $x<c_{L}$ and $y>c_{R}$, then we apply a shift to $(x, y)$. Repeating this as long as such pairs exist, we arrive at a profile $\rho$ that does not contain such a pair. Now $\rho$ either is a central profile, or a left wing profile or a right wing profile. By $(S h)$, we have $F(\pi)=F(\rho)$. If $\rho$ is a central profile, then we are done by the above argument. So assume that $\rho$ is a left wing profile. By Theorems 7 and 10, we have $A M(\pi)=A M(\rho)=\{p\}$. By anonymity, we can write $\rho$ as $\psi^{m} \tau$ such that $\tau$ does not contain a copy of $\psi$. Then, $\rho$ being a left wing profile, $\tau$ is non-empty. If $\tau$ contains $c_{R}$, then $p$ must be odd, so $p=2 k+1$, and $c_{R}=k+1$, and $\tau$ does not contain $c_{L}=k$. For $\rho$ to be a left wing profile, there must be an element $z$ in $\tau$ with $z<k$. By applying a shift to $\left(z, c_{R}\right)$ we get a left wing profile with one occurrence of $c_{R}$ less than in $\tau$. By applying such shifts we get a profile $\zeta$ that does not contain $c_{R}$. By $(S h)$, we have $F(\tau)=F(\zeta)$. Now all elements of $\zeta$ are on $L$. Write $\zeta=\left(y_{1}, \ldots, \ldots, y_{n}\right)$. By $(F 1)$, we have $F\left(y_{j}\right)=\{p\}$, for $j=1, \ldots, n$. By $(C)$, we have $F(\zeta)=\{p\}$. If $m=0$, then $F(\rho)=F(\zeta)=\{p\}$, hence $F(\pi)=\{p\}=A M(\pi)$. If $m>0$, then, by $(C)$ and the case for central profiles, we have $F(\pi)=F(\rho)=F(\psi) \cap F(\zeta)=\{0, p\} \cap\{p\}=\{p\}=A M(\pi)$. The case that $\rho$ is a right wing profile follows similarly. This completes the proof of the theorem.

\section{Independence of the Axioms}

Recall that we always presuppose anonymity to avoid the less appealing usage of multisets. Next we prove that the other five axioms in Theorem 12 are all necessary and form a minimal set of axioms for the characterization of the antimedian function on paths. We list a set of examples which satisfy all five axioms but one, and show that none of the examples is the antimedian function.

To prove consistency below we observe the following. Depending on $A M(\pi)$ we can distinguish between four types of profiles. We call two profiles to be of the same type if they have the same antimedian set. Then the concatenation of two profiles is always a profile of the same type. The concatenation of a balanced profile and an unbalanced profile is of the same type as the unbalanced profile. The concatenation of an extreme profile and an unbalanced profile is of the type of the unbalanced profile. The concatenation of an extreme profile and a balanced profile is again balanced. In all examples below unbalanced profiles of different types will have output sets with empty intersection. So we do not need to check their concatenation for consistency.

To prove the function satisfies $(S h)$, we only need to observe the following. Theorem 8 and Corollary 11 tell us that the shift operation does not change the type of a profile: 
if it is balanced, then it remains balanced, if $A M(\pi)=\{0\}$, then the antimedian set remains $\{0\}$, and if $A M(\pi)=\{p\}$, then the antimedian set remains $\{p\}$.

Example 1. (F1) excluded.

Define the function $F$ on $P$ as follows.

(a1): If $\pi$ is unbalanced, then

$$
F(\pi)= \begin{cases}\{1\}, & \text { when } A M(\pi)=\{0\} \\ \{p-1\}, & \text { when } A M(\pi)=\{p\},\end{cases}
$$

(a2): $F(\pi)=\{0, p\}$ for balanced profiles $\pi$,

(a3): $F(\pi)=V$, for extreme profiles $\pi$.

Due to $(a 1), F$ does not satisfy $(F 1)$. By $(a 2), F$ satisfies $(C e)$, and by $(a 3)$, it satisfies $(E x)$. Note that the output of $F$ is determined by the type of the profile. Shifts do not change the type of the profile, so $F$ satisfies $(S h)$. The observations above on the types of the profiles tell us that $F$ is consistent.

Example 2. (Ce) excluded.

Define the function $F$ on $P$ as follows.

(a4): if $\pi$ is unbalanced, then

$$
F(\pi)= \begin{cases}\{0\}, & \text { when } A M(\pi)=\{0\} \\ \{p\}, & \text { when } A M(\pi)=\{p\},\end{cases}
$$

$(a 5): F(\pi)=V$, for balanced profiles $\pi$,

(a3): $F(\pi)=V$, for extreme profiles $\pi$.

Due to $(a 5), F$ does not satisfy $(C e)$. Due to $(a 4), F$ satisfies $(F 1)$. And due to (a3), $F$ satisfies $(E x)$. Note that again the output of $F$ is defined by the type of the profile. Since shifts do not change the type of a profile, $F$ also satisfies $(S h)$. As above, $F$ is consistent.

Example 3. $(E x)$ excluded.

Define the function $F$ on $P$ by

(a6): $F(\pi)=\{0, p\}$, for extreme profiles $\pi$,

(a7): $F(\pi)=A M(\pi)$, for non-extreme profiles $\pi$.

Due to $(a 6), F$ does not satisfy $(E x)$. Due to $(a 7), F$ satisfies $F(1),(C e)$, and $(S h)$. It is clear that $F$ is consistent.

Example 4. $(C)$ excluded.

Define the function $F$ on $P$ as follows: 
(a8): If $\pi$ is an unbalanced profile with $|\pi| \geq 2$, then

$$
F(\pi)= \begin{cases}\{1\} & \text { when } A M(\pi)=\{0\} \\ \{p-1\} & \text { when } A M(\pi)=\{p\},\end{cases}
$$

$(a 5): F(\pi)=\{0, p\}$, for balanced profiles $\pi$,

(a3): $F(\pi)=V$, for extreme profiles $\pi$.

Moreover, we assume that $F$ satisfies $(F 1)$. Due to $(a 3), F$ satisfies $(E)$. Due to (a5), $F$ satisfies $(\mathrm{Ce})$. Shifts can only be applied of profiles of length at least 2, so $(F 1)$ and $(a 5)$ do not create a problem. For profiles of length at least 2, the output is defined by the type of the profile, so $F$ satisfies $(S h)$. But $F$ is not consistent, because, for any $x<c_{L}$, we have $F(x) \cap F(x)=\{p\}$, whereas $F(x, x)=\{p-1\}$.

Example 5. $(S h)$ excluded.

(a8): If $\pi$ is an unbalanced profile with at least two distinct elements, then

$$
F(\pi)= \begin{cases}\{1\} & \text { when } A M(\pi)=\{0\} \\ \{p-1\} & \text { when } A M(\pi)=\{p\},\end{cases}
$$

(a3): $F(\pi)=V$, for extreme profiles $\pi$,

(a9): $F(\pi)=A M(\pi)$, for constant profiles $\pi$,

(a10): $F(\pi)=\{0, p\}$, for balanced profiles $\pi$ that are not a permutation of $(1, p-1)^{m}$, for any $m>0$, (a11): $F(\pi)=V$, for profiles $\pi$ that are a permutation of $(1, p-1)^{m}$, for any $m>0$.

Due to $(a 9), F$ satisfies $(F 1)$. Due to $(a 10), F$ satisfies $(C e)$. Due to $(a 3), F$ satisfies $(E)$. It is straightforward to check that $F$ satisfies $(C)$. Clearly, $F$ does not satisfy $(S h)$. For instance, applying shifts to $(1, p-1)$ we can get a central profile, which has as output $\{0, p\}$, whereas $F(1, p-1)=V$, due to $(a 11)$.

\section{Axiomatic Characterization of the Antimedian Function on Hypercubes}

In this section, we present a characterization of the antimedian function on hypercubes. The structure of the median sets and antimedian sets in hypercubes is closely related: we can transform the one into the other by taking antipodes. Then using the characterization of the median function on hypercubes from [11], we simply obtain one for the antimedian function. We need a new axiom that is in some sense complementary to betweenness. First we recall some properties of medians and antimedians in hypercubes.

The $n$-dimensional hypercube or $n$-cube $Q_{n}$ has the 0,1 -vectors of length $n$ as its vertex set, two vertices being adjacent if the corresponding 0,1 -vectors differ in exactly 
one coordinate. A vertex $u$ of $Q_{n}$ will be written in its coordinate's form as $u=$ $u^{(1)} \ldots u^{(n)}$. For a vertex $x$ of $Q_{n}$ the vertex $\bar{x}$ is its antipodal vertex, that is, the vertex that is obtained from $x$ by reversing the roles of zeros and ones. Let $X \subseteq V\left(Q_{n}\right)$. Then

$$
\bar{X}=\{\bar{x} \mid x \in X\}
$$

is called the antipodal set of $X$. Obviously, we have $\overline{\bar{X}}=X$.

Let $\pi=\left(x_{1}, \ldots, x_{k}\right)$ be a profile on $Q_{n}$. It is probably part of folklore how to compute the median set of $\pi$ in $Q_{n}$. It can be viewed as a voting per coordinate using majority rule. The median set of $\pi$ in $Q_{n}$ is the subhypercube having 0-s (respectively 1 -s) precisely in those coordinates $j$ for which the 0-s outnumber the 1-s (respectively the 1-s outnumber the 0-s) in the elements of $\pi$. In particular, when $|\pi|$ is odd, $M(\pi)$ is a single vertex. This is a special instance of the Majority Strategy, see e.g. $[9,5,1]$. The antimedian set of $\pi$ is just the antipodal set of the median set: now minority rule is used in the voting process. This was stated as Lemma 2.1 in [1]:

Lemma A Let $\pi$ be a profile on $Q_{n}$. Then $M_{Q_{n}}(\pi)$ induces a subcube of $Q_{n}$. Moreover, $A M_{Q_{n}}(\pi)=\overline{M_{Q_{n}}(\pi)}$.

As observed above, the median function satisfies the three simple and natural axioms $(A),(B)$, and $(C)$. These three simple axioms suffice to characterize the median function on 3-cube-free median graphs, see [5]. Recently it was proved in that these three simple axioms also suffice on hypercubes of any dimension: Theorem 9 in [11]. The proof of this theorem is far from trivial. But with this result in hand, the axiomatic characterization of the antimedian function on hypercubes is now straightforward. We quote the theorem.

Theorem B Let $F$ be a consensus function on $Q_{n}$. Then $F=M$ if and only if $F$ satisfies $(A),(B)$, and $(C)$.

It is a simple exercise to check that, for any two vertices $x, y$ in $Q_{n}$, we have $I(\bar{x}, \bar{y})=\overline{I(x, y)}$. We now introduce the axiom that replaces betweenness.

(CB) Complementary Betweenness: $F(x, y)=I(\bar{x}, \bar{y})$, for all $x, y$ in $Q_{n}$.

Combining Lemma A and Theorem B we get the desired result.

Theorem 13 Let $F$ be a consensus function on $Q_{n}$. Then $F=A M$ if and only if $F$ satisfies $(A),(C B)$, and $(C)$.

Note that also $(C B)$ and $(C)$ are independent. The consensus function $F$ on $V$ defined by $F(\pi)=V$, for all $\pi$, clearly is anonymous and consistent, but does not satisfy betweenness unless $G$ is $K_{1}$ or $K_{2}$. The consensus function $F$ on $V$ defined by $F(x, y)=I(\bar{x}, \bar{y})$ for any $x$ and $y$, and $F(\pi)=V$ for all other $\pi$, satisfies anonymity and complementary betweenness, but not consistency, again, unless $G$ is $K_{1}$ or $K_{2}$. 


\section{Concluding Remarks}

The antimedian function satisfies anonymity and consistency on any metric space. But otherwise one cannot expect much. Only on paths and hypercubes the antimedian function behaves nicely. For hypercubes one other axiom is needed: complementary betweenness $(B C)$. This is due to the non-trivial result that for the median function on hypercubes $(A),(B),(C)$ suffice. Axiom $(B C)$ is defined on hypercubes only. It is not clear how one would generalize this axiom to other classes of graphs, not even to trees or median graphs in general. So this might just be a separate result.

On paths we have four more axioms: one for singleton-profiles, two axioms for individual profiles, that of the center and that of the extreme vertices. Finally, the shift axiom is applicable to all non-central, non-extreme profiles of length at least two. But it still affects only two vertices of the profile. So all axioms are natural and intuitively appealing.

Of course there is one other class on which the antimedian has a very simple axiomatic characterization: the complete graphs. But here the axiom set is trivial: only one axiom is needed, viz. Complement, that is, $F(\pi)$ is the set of all vertices not in $\pi$. So we did not even mention it in the text above. The challenge is to find an axiomatic characterization on other non-trivial classes.

\section{References}

[1] K. Balakrishnan, B. Brešar, M. Changat, S. Klavžar, M. Kovše, and A.R. Subhamathi, On the remoteness function in median graphs, Disrete Appl. Math., 157 (2009), 3679-3688.

[2] R. Holzman, An axiomatic approach to location on networks, Math. Oper. Res. 15 (1990) $553-563$.

[3] S. Klavžar and H.M. Mulder, Median graphs: characterizations, location theory and related structures, J. Combin. Math. Combin. Comput., 30 (1999) 103 - 127.

[4] F.R. McMorris, H.M. Mulder, O. Ortega, Axiomatic characterization of the mean function on trees, Discrete Math. Algorithms Applications 2 (2010) 313 - 329.

[5] F.R. McMorris, H.M. Mulder and F.S. Roberts, The median procedure on median graphs, Discrete Appl. Math. 84 (1998) 165-181.

[6] F.R. McMorris, H.M. Mulder and R.V. Vohra, Axiomatic characterization of Location Functions, Introduction, in: H. Kaul and H.M. Mulder, eds, Advances in Interdisciplinary Applied Discrete Mathematics, Interdisciplinary Mathematical Sciences Vol. 11, World Scientific Publishing, Singapore, 2010, pp. $71-91$. 
[7] F.R. McMorris, F.S. Roberts, C. Wang, The center function on trees, Networks 38 (2001) 84-87.

[8] H.M. Mulder, The interval function of a graph, Math. Centre Tracts 132, Math. Centre, Amsterdam, Netherlands 1980.

[9] H.M. Mulder, The majority strategy on graphs, Discrete Applied Math. 80 (1997) 97-105.

[10] H.M. Mulder, Median Graphs. A Structure Theory, in: H. Kaul and H.M. Mulder, eds, Advances in Interdisciplinary Applied Discrete Mathematics, Interdisciplinary Mathematical Sciences Vol. 11, World Scientific Publishing, Singapore, 2010, pp. $93-125$.

[11] H.M. Mulder and B.A. Novick, An axiomization of the median function on the $n$-cube, to appear in Discrete Appl. Math.

[12] H.M. Mulder, K.B. Reid, M.J. Pelsmajer, Axiomization of the center function on trees, Australasian J. Combin. 41 (2008) 223-226.

[13] R. Vohra, An axiomatic characterization of some locations in trees, European J. Operational Research 90 (1996) $78-84$. 